\title{
Be Relevant (Relevance, Translation and Cross-Culture)
}

\author{
José Mateo Martínez \\ University of Alicante
}

\begin{abstract}
This paper focusses on relevance, translation and cross culture, that is, on the way addressees from different languages and cultures perceive the same messages and how these respond to the varied expectations communicators have and which have been nurtured from a wide cultural experience. Relevance theory offers the right tools to understand the way people from different cultural backgrounds react to the same pieces of information and the effects they cause on them. Using Gutt's application to translation of Sperber and Wilson's "descriptive and interpretive use of language", I highlight the importance of descriptive (covert) translation, not only in examples where the addressee's assumptions must be fulfilled even at the expense of the communicative intentions manifested in the original language but also in other instances where interpretive translation would have been traditionally preferred.
\end{abstract}

\section{Relevance theory, translation and culture}

H.P. Grice (1975) instructed speakers to be relevant in their speech that is to offer the information they consider more important or relevant for their hearers. This, evidently, logical and harmless statement was the starting point of Sperber and Wilson's (S\&W, henceforth) Relevance Theory, one of the most innovative proposals for the understanding of language and the cognitive and linguistic procedures that organize communicative 
interaction. However, S\&W's approach has also been controversial. ${ }^{1}$ For example, this view of communication has been accused of restricting its scope only to one-to-one interaction ignoring social and cross-cultural issues (Harvey, P. 1988). And even there S\&W have been blamed for overemphasizing the importance of the decoding efforts made by the recipient of the utterance while showing little interest for the communicator or language user (J.L. Mey \& M. Talbot, 1989). Most of this criticism has been refuted not only by $S \& W$ themselves but also by many other researchers who believe Relevance Theory has a lot to offer in the understanding of human communication.

This paper is about translation. Therefore it is about language, communication and cross-cultural transfer, ${ }^{2}$ seen from the cognitive approach of Relevance Theory. This perspective can offer translators important keys in order to understand some of the cognitive processes employed to transfer not only meanings but also intentions from one language into another. As we well know, this is a fundamental component in communication that has not always been taken into account by many translators in their work. Not much research has been devoted either to relevance and translation except for E-A. Gutt's classic text (1991) and a handful of papers. Perhaps the reason is that little theoretical work in translation was done at all in the past when most efforts were wasted discussing the old aphorism "traduttore traditore" which questioned altogether the very existence of translation. Fortunately, this situation changed in the late 70 s and early 80 s and many interesting theoretical works have been written since then, ${ }^{3}$ Among the different ways to approach translation, I will consider it as an act of communication which must be explained through communication models. If we concede that the old code model has been surpassed by other accounts, then we are left with Relevance Theory.

Basically, Sperber and Wilson's Theory of Relevance $(1986,1995)$ focusses on the mental mechanisms which allow participants in conversations to process in the most effective way and with the smallest effort the information that flows from speakers to hearers. They explain relevance as:

\footnotetext{
"A property of inputs to cognitive processes. The processing of an input (e.g. an utterance) may yield some cognitive effects (e.g. revisions of beliefs). Everything else being equal, the greater the effects, the greater the relevance of the input. The processing of the input (and the derivation of these effects) involves some cognitive effort. Everything else being equal, the greater the effort, the lower the relevance" (Sperber and Wilson, 1997: 115)
}

Inference is the key process in achieving this objective. This cognitive ability allows the hearer to recognize the speaker's informative and communicative intentions. But S\&W's view of this phenomenon is far reaching. It not only accounts for the implications and intentions covered by the actual words uttered by the communicator but also for those meanings which are impossible to encode verbally. Inference is helped by other fundamental elements in comprehension, i.e. context and shared or mutual knowledge. Although it seems relatively easy to analyse the implicatures and meanings conveyed by speakers in their utterances especially if we have access to additional contextual and 
cotextual information, the description of the cognitive processes of inference used by hearers to decode the linguistic input and the nonlinguistic information is still an issue under strong debate especially among philosophers, psychologists and discourse researchers. An utterance seems to be, after all, a "finished" product that conveys the speaker's definite set of intended meanings and ostensive stimuli and which is physically perceived and mentally interpreted by the activation of specialized mental areas of our brain.

Relevance Theory believes that the inferential nature of comprehension has a universal character, that is, it is shared by all human beings. Inference is mainly used to solve the problem of the existence of more concepts than words can describe. It is only through inference that, addressees can:

a) Isolate and categorize the different meanings that can account for a satisfactory interpretation of an utterance at a conceptual and cognitive level. (Different concepts for the same words).

b) Choose, with the aid of additional information, (context, mutual and encyclopaedic knowledge, paralinguistic recourses) the concept that best responds to their communicative interests and apply it to the utterances they hear.

Pragmatic comprehension is a cognitive mechanism whose processes are not fully understood and therefore have not been definitely explained yet, in spite of such appealing models as Chomsky's competence or Fodor's modules that describe human minds as composed of central and modular systems, the first being of a more abstract nature while the second would respond to external stimuli, ${ }^{4}$ Sperber (1996a: 119-150, 1996b:14) himself advocates a modular image of the mind to explain not only language use but also culture. ${ }^{5}$

However, and in spite of the assumed universal character of inference (based on identical systems of cognitive processing and utterance-decoding for all languages), there is also a more individualistic side in the way we access and perceive meanings. It is a personal response determined by psychological (mood, attention, interest, etc.) and sociological reasons (language used, cultural and national idiosincracy). This second aspect of inference is more evident in the case of communicative relevance ${ }^{6}$ and can lead to a wide range of interpretive possibilities when we apply it to translation. The perception of relevance by an addressee in a communicative setting where a communicator ostensively addresses a message to him can activate different inferential processes depending on whether the recipient speaks the same or a different language or whether he uses similar or different cultural patterns. In this context the speaker/writer may express concepts and ideas which will carry a different informative load depending on the addressee's language and cultural experience. Implicatures will then vary and so will the way the receiver/reader anticipates meanings and searches for a relevant interpretation.

Besides this intimate character of comprehension, there are also social patterns of inference, practically shared by all members of the same community, where expectations on certain messages and utterances are similarly grounded. We can then agree that the contextual effects of an utterance or sentence produced in a given language will vary 
profoundly if it is listened to or read by listeners or readers whose native language is different and with different expectations. In consequence, implicatures, ostension, linguistic and pragmatic features, the strengthening or weakening of the hearer's inferential processing of the original message will have to comply, as we will see further on, with his linguistic and communicative expectations.

\section{The translation of cognitive and cultural information}

Translation means, among other things, comparing and contrasting two different linguistic and cultural systems. Translators try to satisfy the cognitive expectations of their ST (source text) readers. To accomplish this target, they must overcome the problem of transferring a message to an alien context which contains meanings that were originally thought and addressed to a reader who shared with the addressor the same linguistic, pragmatic and cultural world. The cognitive stimuli displayed in translations (context, shared-knowledge, concepts and other cultural hints) aim to ease the TT (target text) reader's understanding and achieve a similar cognitive response. On reading a translation it is expected that readers will set up the hypotheses of meaning that best answer their expectations and which fit the ST communicative intentions. It is very likely that many cognitive stimuli will not be correctly interpreted by the TT addressee if the translator fails to transfer not only the linguistic and semantic meanings of the original text but also the underlying cognitive framework which accounts for the exclusive vision of the world and cultural layout shown in the source language. In S\&W's words, the messages issued by the communicators are absorbed by the hearer's cognitive environment where all facts "that he can perceive or infer: all the facts that are manifest to him abide" (S\&W, 1986:39). By comparing this cognitive environment with his physical milieu (context, time, social organization, etc.) the ST addressee has a clear advantage over the TT addressee when he generates meaning predictions or conceptual representations of the world which normally match those of the sender's.

The translator's task includes the generation of presumptions of relevance in the target language similar to the ones originated by the source message. It is crucial that the communicative goals achieved between the ST author and the TT reader should coincide with those experienced by the ST reader. As these presumptions do not often come out the same when they are transferred from one language to another, a process of adaptation must take place so that similar cognitive effects and inferences are reached and the intended meaning is understood. TT readers are asked to adapt their expectations to a new cultural environment. Therefore they can build new assumptions in the light of the contextual effects they receive with the translated text. Readers try then to process the new information. This process can take place rapidly or slowly depending on the amount of new input they can filter through their previous experience until it becomes meaningful and in accordance with their world knowledge. Translators must try to make this interpretive flow as effective as possible. 
The notion of context in RT is somehow restricted and applies mainly to the addressee, that is, in the way he is constantly comparing the new information to that stored in his longtime memory with the purpose of retrieving adequate contextual effects which will allow him to match his inferred meanings to the speaker's underlying intentions. This personal view of context is, nevertheless, one part of a macro context regulated by social expectations and knowledge. These account for all those experiences that are shared by most if not all speakers in the same community who tend to respond similarly to the stimuli contained in certain types of messages (advertising, politics, etc.)

Translators usually work with written texts ( $\mathrm{I}$ am not considering oral translation in this paper, which I reckon is a fascinating field of research from the RT perspective). Their work has been traditionally viewed as an effort to convey the words, style and intentions expressed by authors in a ST to their potential TT readers as faithfully as they can. This is a process in which the translator is seen to act as a kind of go-between who bridges the cultural, linguistic and communicative gaps between author and reader and who is finally responsible for the success in the way the reader infers the right assumptions and all the contextual effects of the original meaning. But again, the readers' cultural roots which imply aspects such as mutual knowledge, context and ostensive linguistic and paralinguistic features, will not always match those of the author's and will have to be modified or adapted to make them accessible to the reader. The main objective for the translator should be to ensure that the balance between the contextual effects and the processing efforts deployed by his TT reader is equivalent to the balance achieved by the ST reader. That objective raises one problem: how can the translator know that the resulting effect of the text in the TT reader is similar to the effect produced in the ST reader? (Gutt, 1991:48) Answering this question does not necessary imply that the translator is a source language native or a perfect bilingual (including a deep source language world and encyclopaedic knowledge) which, as we know, is not usually the case. Since a cognitive representation of translated meanings cannot be an exact replica of the cognitive representation of original meanings, translators must try to obtain equivalent responses from their readers by interpreting and adapting one code to another and where "equivalence" is more important than "accuracy."

Translators are commonly TT natives with a good command of the languages they translate, who may or may not live in the source language country and therefore be or not be acquainted with all the communicative and social aspects of this language. This opens up a different perspective of the case. Since translators work for the text receiver, they focus their work not on the ST reader's interpreting efforts but on those required of the TT reader. Translators must process the original text and infer the right meanings (in fact, they should act exactly like a native reader). Once this has been done, they should start modelling the TT according to their potential readers' expectations with the aim of producing a message which, having included the original assumptions, manages to fulfill these expectations from a cognitive, linguistic and social perspective. 


\section{Descriptive and interpretive translation}

Gutt's distinction between descriptive and interpretive translation (1991:56-65) is very suitable in order to understand the translation process as a communicative act. The translator's role varies in both cases: if we consider the interpretive translation first, we see it includes, as faithfully as possible, the speaker's communicative intentions often wrapped in a text form which resembles that of the ST. The translator is the vehicle used by the author to reach the TT recipient. Literary and scientific translations would be proper examples of interpretive translation where resembling the original thought and the linguistic, stylistic and semantic layout seems to be paramount. However, there is a different type of translation Gutt defines as "covert" or descriptive translation ${ }^{7}$ which works differently. The translator gathers the ST communicative intention by means of extracting relevant information and moves it into the TT making all the necessary adjustments so that the resulting text fulfills the reader's expectations. In this process he may change ST cognitive stimuli by including TT ones. He can add new contextual effects, meanings, cultural data, etc. He can modify the text to such an extent that it will no longer resemble the ST it originally came from. The real intention behind this procedure is not to reduplicate the ST communicative effects, which are not likely to achieve the same cognitive results in the TT recipient, but to adapt those effects to the intentional and cultural expectations held by the TT addressee in order to get a "similar" response.

The translator believes that the cultural constraints between the two languages are so important that he would have never obtained the same contextual effects in his TT readers ${ }^{8}$ had he used only an interpretive translation of the ST. I would like to comment on this point with the following case of cosmetic publicity:

"After a shower or bath, gently massage Revitalizing Body Lotion all over the body. Used regularly it leaves the skin satin-soft."

"Aplicar la leche Cuerpo Sedoso en amplios y ligeros masajes por todo el cuerpo, preferentemente tras el baño o la ducha. Su aplicación regular asegura una piel suave, firme y joven." 10

This example of textual transfer from English into Spanish shows that the translator opted for a more descriptive than interpretive translating strategy. But, as Gutt puts it, is it really a translation? or is it just a new text with different communicative intention? In my opinion, it is a translated text, because the general meaning is identical: it keeps the original ST communicative intention and recipients of both languages will perform similar cognitive efforts by which they will infer the meanings sought by the communicator (the cosmetics manufacturer). If the requirements of relevance in both messages (intention + ostension + contextual effects + inference + assumptions...) are equally realized, then the differences between them can be explained in terms of interlanguage and cross-cultural communication instead of the cognitive effort involved. In short, this type of text would preserve a distant 
interpretive relation (same original assumptions and explicatures demanding equal cognitive response) but in a typically descriptive format: the original text has been deeply modified in the translation process.

The English text shows a weighted balance between intention, ostension and information. Its purpose is to get an adequate cognitive response and contextual effects that make its message relevant according to the English reader's assumptions. However, a more "interpretive" translation into Spanish (see note 9) would not achieve the same objective. Apparently Spanish readers of cosmetics (and many other types of) advertisements require more informative input. In order to believe what the advertiser says, cognitive stimuli must be stronger, intentions more ostensive and the contextual effects must fulfill the readers' almost miraculous expectations. This different cultural perspective should be accounted for by the translator. In fact the manufacturer's intention is not to say what the product is about and what it does but to sell it. To do that it is extremely important that the resulting message complies with what the TT readers might expect of such messages. Thus, the need for linguistic, pragmatic, cognitive and social adjustments makes the use of descriptive translation necessary in this case. On the other hand, a literal translation into English of the Spanish text might prove ineffective for most English readers who would consider its overemphasis and exaggeration inadequate, distorting and what is worse, untrue.

Furthermore, the recipient's stimulation of his comprehension mechanisms depends on the activation of his encyclopaedic knowledge on the issue. This knowledge establishes his set of expectations about what he is about to read. Therefore, The Spanish reader expects specific kinds of messages with specific layouts, different from those expected by the Anglo Saxon reader who reads similar messages. If we believe that different degrees of relevance are necessary for communicating the same message in different languages, then we might as well consider the idea of a contrastive or cross cultural layer in the principle of relevance. In it, the explicitness of ostensive communication would be determined by the message's cultural and idiosyncratic character.

As a consequence, I believe that we cannot trace clear limits between what Gutt defines as descriptive or "incidental" (1990:142) (covert) translation and interpretive (real) translation (1990, 1991). I would rather think of a translating continuum that ranges from translations that, originating in an SL idea, make a TT that bears little resemblance to the original ST but which manages to convey a similar presumption of relevance to texts that keep all ST assumptions but fail to produce similar cognitive efforts in the TT readers because they maintain an excessively literal format. In between we can find translations with different degrees of descriptive and interpreting content and therefore different levels of communicative relevance for their recipients. The translator can, for instance, make translations which are "faithful" to the ST, or opt to keep some "flavour" of the ST by translating only a few ST reader-oriented sentences while maintaining the rest TT-readeroriented. ${ }^{11}$ Within this framework, there can be a flow of communication where translated texts can be sometimes descriptive and at other points interpretive depending on their readers' expectations but without marking off clear boundaries between the two. 
On the other hand, the linguistic codes used in utterances or texts (no matter how clear, precise and well expressed they may be) are not always capable of communicating the speaker/writer's real intentions because, as I have mentioned above, the world of concepts, intentions and meanings is far richer than the world of words. As a consequence, translations will have a higher interpretive tone if they focus on the way (utterances, sentences, implicatures, ostension, etc.) addressors have organized their messages. But these translations will be considered to be descriptive if they extract the underlying intentions and meanings to adapt them to the recipients' expectations by modifying both the ST's cognitive stimuli (cultural patterns, world and shared knowledge, etc.) and text format (word and semantic adjustments, added sentences and so on). As a result, a descriptive translation will have a more evident cognitive nature: ST ideas will have been inferred and translated into equivalent TT ones but using different linguistic and semantic units and cultural settings. On the other hand, an interpretive translation will have a more linguistic character: ST linguistically-expressed ideas will have been translated into equivalent TT linguistically-expressed ones. But in all cases, every time an original thought or idea needs to be communicated in another language, we can talk of translation. This assertion should not give the impression that I advocate a sort of translation practice where the resulting text is always subordinated to the underlying intention regardless of the way this intention has been manifested in the source language. What I mean is that a translator must first understand the intentions and cognitive relevance of the message to be translated and then look for the adequate linguistic and cultural ways to make them accessible to target language recipients. This procedure includes not only a functional but also a formal approach.

Let me illustrate these words with another piece of advertising copy where the translator has rendered both a descriptive and an interpretive translation. In a Kenwood (Hi-Fi equipment manufacturer) advert for a new music system, the writer's primary intention is to present a fully-equipped Hi-Fi system at a reasonable price. To achieve this goal, he opens the advert with the following line:

\section{"List of features as long as your arm"}

Then we can see a picture depicting a sophisticated Hi-Fi set with several speakers and other electronic items. Just below the picture another line reads:

\section{"But not an arm and a leg"}

The text combines literal (first sentence) and figurative (second sentence) meanings playing a witty game of analogies. The first line emphasizes the manufacturer's intention of showing a very complete piece of equipment where nothing one could wish for is missing and even more than expected is being offered. Ostensive stimuli include words and a picture. The second sentence prevents the reader from inferring the wrong contextual effect of believing that such complete equipment must be "necessarily" expensive. Using an idiomatic sentence 
(meaning "not very expensive") readers access the intended meaning: a not-very-expensivefully-equipped-music system.

The translation into Spanish of this advert has the same format (including the same picture) but its lines read differently:

"Abra bien los ojos y descubra todas sus prestaciones"

(Open your eyes wide and discover all its features)

"Pero sin costarle un ojo de la cara"

(But that won't cost you one of your eyes)

The Spanish version keeps the original intention but has changed the linguistic output to achieve equivalent cognitive effects. By using similar linguistic resources (literal and figurative language) and similar ostensive stimuli (picture + language) the TT manages to create the same contextual effects and an identical cognitive effort through the correct transfer of linguistic and cultural data. The translator has translated the text in an interpretive manner: similar linguistic uses convey the intended idea and create the same contextual effects and the same inferential processes in both ST and TT readers. And he has also used a descriptive approach by adapting language and intentions to the recipients' expectations.

In general, utterances try to stimulate the hearer's attention from two perspectives ( $R$. Carston, 1997): ostensively by which the hearer's attention is captured and then reacts trying to interpret the message and linguistically by means of a code where meanings are transported and where the reader's inferential processing is justified. The speaker must be sure of two things:

a) his message includes enough cognitive effects so that the receiver perceives it is worth decoding, and;

b) the receiver should not have to make an excessive effort to access those effects.

The Kenwood advert complies with both conditions in English and in Spanish so the ultimate intention is easily accessed by most readers of these two languages. Both adverts have used practically the same amount of linguistic data to reach the intended goal. In the case of the cosmetics advert studied before, both versions also fulfill the requirement of offering enough ostensive stimuli to make the communicative act possible, and, following S\&W, to attract the recipient's attention and guide this attention to the author's real intentions with the purpose of making them explicit, although the linguistic changes made along the way have been profound and important (the Spanish text needed to expand the quantity and quality of its input to achieve similar results to the ones in the English text).

It is generally assumed that advertising plays with both ends of relevance. Very often it strives to offer as many ostensive stimuli as possible so relevance is big and the receiver's 
inferring effort is small (strong implicatures). In other cases, however, there is a minimum level of ostension which requires a big cognitive effort in the receiver's readiness to infer the right meaning (weak implicatures). There is a swinging movement from maximum to minimum ostension. It is fairly rare to find the balanced-point which is more common in many conversational exchanges. In the cosmetics advert, for example, we would be in the first case: maximum ostension, maximum relevance, minimum inference, minimum processing effort and maximum contextual effects. Due to the higher degree of ostensive input in the Spanish version one might be tempted to think that it is more relevant than the English advert. We might also believe in the existence of other collateral effects that could result in an increase in the Spanish reader's comprehension effort and, therefore, in the process of inferring meanings. And yet this is not the case because these effects would be minimized by this Spanish reader's cultural and idiosyncratic awareness.

\section{Notes}

1. As well-known examples of this, I can mention the reviews of the first version of Relevance, written by Levinson and J.L. Mey \& M. Talbot in 1989. Levinson criticized what he considered to be a simplistic approach to communication and S\&W's attempt to reduce pragmatics to "a single cognitive principle, a mental reflex" (469). J.L. Mey \& M. Talbot objected in their review article to the user's disappearance in S\&W's book, whose role had been reduced to be a "lending personae to the author's examples and illustrating sketches" (279). S\&W's sociological and psychological beliefs have also been considered to be reductionist, an opinion shared by Gorayska, B and R. Lindsay (1993).

2. By culture I understand first what Gumperz (1993:206) defines as "cultural knowledge" that is "knowledge of the world acquired primarily through locally (regionally or nationally, my italics) based personal experience, through face to face (or education, my italics) interaction." It is a personal or individual notion of culture which relates to private world knowledge. But I also reckon this is just one side of the coin; the other should include aspects such as group beliets, values and symbols that the translator should also account for. For the purpose of this paper, however, I focus on a more face to face cultural encounter: a source text that is translated into a target text and which is read by individual readers with different language and cultural knowledge. Obviously this knowledge can be (and actually is) shared by many other individuals within the same nation who have had access to the same or similar cultural experiences.

3. Although in several countries, like Spain, translation has been only recently included in the university curriculum and is still considered in some bastions of linguistic research as a craft with no scientific background.

4. In its functioning, Fodor (1983) carefully distinguishes between aspects that can be understood and analized (modular systems) and aspects which are so complex that they cannot be understood (central systems).

5. In his interesting Times Literary Supplement article, he writes: "If the mind is modular, if it is highly selective in the inputs it attends to, and highly biased in the kinds of inference it draws from these inputs, if, moreover, attention, inference and learning proceed in a great variety of ways, each tailored to a specific domain of information, then psychology should be a 
crucial resource in explaining culture"(1996:15).

6. Sperber and Wilson in the updated 1995 edition of their classic work Relevance split their original Principle of Relevance into two separate principles:

Cognitive Principle of Relevance: Human cognition tends to be geared to the maximisation of relevance (to achieving as many cognitive effects as possible for as little cognitive effort as possible); and

Communicative Principle of Relevance: Every act of ostensive communication communicates a presumption of its own relevance (appropriate ostensive effort to justify the expected decoding effort)

7. In fact, Gutt (1991:121) considers descriptive translation as covert or non translation.

8. I am not only talking of the contextual effects determined by the cultural assumptions shared by most TT readers, but also include the individual reactions which may interpret the same message differently depending on psychological mood or sociological status, even within the same language

9. The literal translation in Spanish would be: "Después de la ducha o el baño, aplíquese la loción Cuerpo Revitalizado por todo el cuerpo con suavidad. Usada con regularidad deja la piel sedosa."

10. The literal translation in English would be: "Apply Silky Body milk in a thorough, gentle massage all over the body, preferably after a bath or shower. Used regularly it will ensure a smooth, firm, young skin."

11. This strategy is put into practice by M. Hjort-Pedersen (1996) in her paper on the translation of wills from Danish into English.

\section{Works cited}

Blakemore, D. Semantic Constraints on Relevance. Oxford: Blackwell, 1987.

Fodor, J. The Modularity of Mind. Cambridge, MA: MIT Press, 1983.

Carston, R. "Relevance-theoretic pragmatics and modularity." UCL Working Papers in Linguistics 9 (1997): 29-53.

Carston, R. \& S. Uchida, eds. Relevance Theory. Applications and Implications. Amsterdam: John Benjamins, 1998.

Cummings, L. "The scientific reductionism of relevance theory: The lesson from logical positivism." Journal of Pragmatics 29 (1998): 1-12.

Gorayska, B. \& R. Lindsay. "The roots of relevance." Journal of Pragmatics 19 (1993): 301-323.

Grice, H.P. "Logic and Conversation." Speech Acts (Syntax and Semantics 3). Eds. P. Cole and J. Morgan. New York: Academic Press, 1975. 41-78.

Gumperz, J. "Culture and conversational inference." Trends in Linguistics. Studies and Monographs 69. The Role of Theory in Language Descriptions. Ed. W. Foley. Berlin: Mouton de Gryter, 1993.

Gutt, E-A. "A Theoretical Account of Translation Without a Translation Theory." Target 2:2 (1990): 135-164.

Gutt, E-A. Translation and Relevance: Cognition and Context. Oxford: Blackwell, 1991.

Harvey, P. "Relevance Theory and Anthropology." Liverpool Papers in language and Discourse, 
$1(1988): 50-63$.

Higashimori, I. \& D. Wilson. "Questions on Relevance." UCL Working Papers in Linguistics, 8 (1996): 111-125.

Hjort-Pedersen, M. "Legal translation and the principle of relevance." Multilingua 15/4 (1996): 361-371.

Levinson, S. "A review of Relevance." Journal of Linguistics 25 (1989): 455-472.

Mey, J. L \& M. Talbot. "Computation and the soul." Cognitive Aspects of Language Use. Ed.

A. Kasher. Amsterdam: Elsevier Science, 1989. 239-285.

Pilkington, A. "Poetic effects." Lingua, 87 (1992): 29-51.

Sperber, D. Explaining culture: A Naturalistic Approach. Oxford: Blackwell, 1996a.

Sperber, D. "Learning to pay attention: How a modular image of the mind can help to explain culture." Times Literary Suplement, December 27 (1996b): 14-15.

Sperber, D. and D. Wilson. Relevance: Communication and Cognition. Oxford: Blackwell, $1986 / 1995$.

Sperber, D. and D. Wilson. "The mapping between mental and public lexicon." UCL Working Papers in Linguistics 9 (1997): 111-125. 